\title{
Deposição de ácido linoléico conjugado (CLA) em tilápias-do-nilo
}

\author{
Lilian Dena dos Santos ${ }^{1}$, Wilson Massamitu Furuya ${ }^{2}$, Makoto Matsushita ${ }^{3}$, Lilian Carolina \\ Rosa da Silva ${ }^{2}$, Tarcila Souza de Castro Silva ${ }^{2}$, Daniele Botaro ${ }^{4}$, Priscila Pinsetta Sales ${ }^{2}$ \\ 1 Bolsista da CAPES; Doutoranda, Programa de Pós-graduação em Zootecnia, Universidade Estadual de Maringá - UEM, Av. Colombo, 5790, \\ Maringá - $P R$. \\ 2 Departamento de Zootecnia-UEM, Maringá - PR. \\ 3 Departamento de Química-UEM, Maringá - PR. \\ 4 Pós-graduação em Biofísica Ambiental, IBCCF - Universidade Federal do Rio de Janeiro.
}

RESUMO - Objetivou-se avaliar a deposição de ácido linoléico conjugado (CLA) em filés de tilápia-do-nilo (Oreochromis niloticus). Foram utilizados peixes com 121,08 $\pm 8,48 \mathrm{~g}$, distribuídos em quatro caixas de cimento amianto $\left(1,0 \mathrm{~m}^{3}\right.$ cada uma) e arraçoados até saciedade aparente, duas vezes ao dia, durante 49 dias. Como alimento, utilizou-se ração comercial extrusada com $28 \%$ de PB e $3.000 \mathrm{kcal}$ de energia digestível $/ \mathrm{kg}$, acrescida de $2 \%$ do produto comercial Luta-CLA ${ }^{\circledR}$ (BASF - Brasil). No início do experimento, cinco peixes foram coletados aleatoriamente como amostra controle (sem CLA). A cada sete dias, dois peixes de cada caixa foram coletados, pesados e filetados para determinação da taxa de deposição de CLA nos filés. As amostras de filés foram estocadas a $-24^{\circ} \mathrm{C}$ para análise do teor de lipídios totais e do perfil de ácidos graxos. A taxa de deposição de CLA foi determinada pela função de Gompertz $(\mathrm{y}=\mathrm{a} \cdot \exp \{-\mathrm{b} . \exp [-\mathrm{kt}]\})$. O meio-tempo (T) para deposição de $50 \%$ do CLA foi estimado pela equação $\mathrm{T}=\ln 2 / \mathrm{k}$, em que: $T$ representa o meio-tempo; $\ln$, o logaritmo Neperiano e $k$, a unidade de tempo que fornece a "velocidade" de deposição do CLA no tecido muscular da tilápia-do-nilo. A deposição máxima de CLA nos lipídios totais do filé de tilápias-do-nilo estimada pela função de Gompertz foi de 1,61 g/100 g de lipídios do filé. O meio-tempo de deposição de CLA nos lipídios do filé ocorre com 6,86 dias.

Palavras-chave: ácidos graxos, ácido linoléico conjugado, Oreochromis niloticus

\section{Deposition of conjugated linoleic acid (CLA) in Nile tilapia}

\begin{abstract}
This study was carried out to determine the deposition of conjugated linoleic acid (CLA) in Nile tilapia Oreochromis niloticus fillet. Fish averaging $121.08 \pm 8.48 \mathrm{~g}$ were distributed in four cement amianthustank $\left(1.0 \mathrm{~m}^{3}\right.$ each $)$ and hand fed to apparent satiation, two times a day for 49 days. As feed, an extruded commercial diet with $28 \%$ CP and $3000 \mathrm{kcal}$ of digestible energy was utilized. A commercial CLA product (Luta-CLA ${ }^{\circledR}$, BASF) was added at $2 \%$ of the diet. At the beginning of the experiment, five fish were randomly collected as a control sample (without CLA). Weekly, two fish of each tank were collected, weighed and filleted for measurement of CLA deposition ratio in the fillets. The fillet samples were stored at $-24^{\circ} \mathrm{C}$ for total lipid and analyses of fatty acids profiles. The deposition rate of CLA was determined by Gompertz function $(y=a \cdot \exp \{-b \cdot \exp [-\mathrm{kt}]\})$. Half time $(\mathrm{T})$ to have $50 \%$ deposition of CLA was calculated by the equation $\mathrm{T}=\ln 2 / \mathrm{k}$, where $T$ is half time; $\ln$ is Napierian logarithm and constant $k$ the time unit, which provides the "velocity" in the deposition of CLA in the Nile tilapia fillets. The maximum CLA deposition in the fish fillet lipids, estimated by the Gompertz function, was $1.61 \mathrm{~g} / 100 \mathrm{~g}$ fillets lipids. Half time of CLA deposition in the fillet lipids occur within 6.86 days.
\end{abstract}

Key Words: conjugated linoleic acid, fatty acids, Oreochromis niloticus

\section{Introdução}

Entre os setores da produção animal, a aqüicultura é o que se expande mais rapidamente. A tilápia-do-nilo (Oreochromis niloticus) é uma espécie de rápido crescimento e possui carne com boas características sensoriais e filés sem espinhos intramusculares, o que a torna uma das espécies mais cultivadas em criação intensiva em regiões tropicais e subtropicais.
Ácido linoléico conjugado (CLA) é um termo utilizado para designar um grupo de ácidos octadecadienóicos, que são isômeros conjugados posicionais e geométricos do ácido linoléico (C18:2) em que as duplas ligações são separadas por uma ligação simples carbono-carbono no lugar de um grupo metileno, dos quais o cis-9,trans-11 e otrans-10,cis-12 possuem atividades biológicas (Pariza et al., 2001). O 18:2 (cis-9,trans-11) é considerado a forma primária de CLA presente naturalmente nos alimentos, 
ainda que o 18:2 (cis-9,trans-11) e o 18:2 (trans-10,cis-12) sejam os dois isômeros predominantes e presentes em níveis semelhantes no CLA sintético (Chin et al.,1992).

A utilização de CLA tem sido objeto de pesquisas sobre ácidos graxos, em virtude de seus benefícios à saúde humana (Whighamet al., 2000). O fornecimento de CLA em dietas para suínos, aves e peixes tem melhorado suas características de produção (Park et al., 1997; Ostrowska et al., 1999; Twibell et al., 2000; Yang et al., 2002).

Existem evidências de que os isômeros cis-9,trans-11 e trans-10, cis-12 CLA podem agir beneficamente em sistemas biológicos, mas de forma diferente. Os efeitos do fornecimento de CLA em dietas para peixes foram avaliados durante 60 dias por Choi et al. (1999), citados por Ackman (1999), na carpa-comum (Cyprinus carpio), em tilápias-do-nilo e rockfish (Sebates schlegi). Twibell et al. (2000) estudaram híbridos de striped bass (Morone chrysops $\times$ M. saxatilis) alimentados com 1,0 g/100 g CLA durante 60 dias, enquanto Twibell et al. (2001) realizaram estudo com juvenis de perca-amarela (Perca flavescens) alimentados com 0,5 e 1,0 g/100 g CLA durante 67 dias de experimento.

Diversos fatores podem influenciar a taxa de deposição de lipídios corporais, uma vez que a habilidade dos peixes em acumular CLA depende da espécie e do tipo de lipídios da dieta (Twibell et al., 2001), sendo importante determinar o número de dias necessários para a incorporação de CLA nos lipídios do tecido muscular.

Este trabalho foi realizado com o objetivo de determinar a taxa deposição de ácido linoléico conjugado (CLA) nos lipídios totais do filé de tilápias-do-nilo.

\section{Material e Métodos}

O experimento foi realizado no Laboratório de Aquiicultura durante o período de dezembro de 2004 a janeiro de 2005, durante 49 dias.

Foram utilizados 56 peixes revertidos, com 121,08 $\pm 8,48 \mathrm{~g}$ de peso vivo (PV), originados da Piscicultura Araucária Belmonte, Rolândia-PR, distribuídos em quatro tanques de cimento amianto com volume unitário útil de $1,0 \mathrm{~m}^{3}$, em densidade de estocagem de 14 peixes por tanque.

Em cada tanque, foi instalado sistema de aeração com difusores acoplados a um soprador central, de modo que o oxigênio dissolvido foi mantido entre 4 e $6 \mathrm{mg} / \mathrm{L}$. A temperatura da água foi mantida em torno de 24 a $28^{\circ} \mathrm{C}$ por meio de aquecedores (150 watts). A retirada das fezes foi realizada por meio de sifonagem diária dos tanques e a renovação diária de água foi de $20 \%$. Os tanques foram cobertos com lona plástica para minimizar a produção primária.

Os peixes foram alimentados com dieta comercial extrusada (6 mm de diâmetro) com $28 \%$ de PB, 3.000 kcal de energia digestível/kg e 3,25\% de lipídios totais (Tabela 1).

Tabela 1 - Composição da dieta experimental (\%)

Table 1 - Composition of the experimental diet (\%)

Ingrediente

$(\%)$

Ingredient

Milho

Corn 22,85

Gérmen de milho

Corn germ

Farelo de trigo

30,00

Wheat meal

Farelo de soja

8,00

Soybean meal

Farinha de carne

3,00

Meat meal

Farinha de peixe

1,00

Fish meal

Farinha de vísceras de frango

25,00

Poultry by-product meal

Calcário calcítico

Limestone

Suplemento mineral e vitamínico ${ }^{1}$

0,50

Mineral and vitamin mix

Vitamina $\mathrm{C}^{2}$

0,03

Vitamin C

Sal

0,50

Salt

MS, $\%^{4}$

$D M$

Energia digestível $(\mathrm{kcal} / \mathrm{kg})^{5}$

$3.036,13$

Digestible energy

$\mathrm{PB}, \% 4$

$C P$

$\mathrm{FB}, \% 4$

CF

Lipídios totais $(\%)^{6}$

6,63

Total lipids

$\mathrm{Ca}(\%)^{4}$

$\mathrm{P}$ disponível $(\%)^{5}$

2,47

0,76

Available $P$

Ácido linoléico conjugado (g/100 g de lipídios $)^{6}$

Conjugated linoleic acid ( $\mathrm{g} / 100 \mathrm{~g}$ lipids)

1 Suplemento mineral e vitamínico (Mineral and vitamin mix) (Supremais Campinas-SP): composição por kg do produto (composition perkg the product): vit. $A=1200.000 \mathrm{UI}$; vit. $\mathrm{D} 3=200.000 \mathrm{UI}$; vit. $\mathrm{E}=12.000 \mathrm{mg}$; vit. $\mathrm{K} 3=$ $2.400 \mathrm{mg}$; vit. $\mathrm{B} 1=4.800 \mathrm{mg}$; vit. $\mathrm{B} 2=4.800 \mathrm{mg}$; vit. $\mathrm{B} 6=4.000 \mathrm{mg}$; vit. $\mathrm{B} 12=4.800 \mathrm{mg}$; ác. fólico $($ folic $a c i d)=1.200 \mathrm{mg}$; pantotenato de cálcio (calcium pantothenate) $=12.000 \mathrm{mg}$; vit. $\mathrm{C}=48.000 \mathrm{mg}$; biotina (biotin) $=48$ $\mathrm{mg}$; colina $($ choline $)=65.000 \mathrm{mg} ;$ niacina $($ niacin $)=24.000 \mathrm{mg} ; \mathrm{Fe}=10.000$ $\mathrm{mg} ; \mathrm{Cu}=600 \mathrm{mg} ; \mathrm{Mg}=4.000 \mathrm{mg} ; \mathrm{Zn}=6.000 \mathrm{mg} ; \mathrm{I}=20 \mathrm{mg} ; \mathrm{Co}=2 \mathrm{mg}$; $\mathrm{Se}=20 \mathrm{mg}$;

2 Vitamina C: sal cálcico 2-monofosfato de ácido ascórbico com $(42 \%$ de princípio ativo) (calcium salt, ascorbic acid 2-monophosphate - $42 \%$ active principle. 3 Butil hidroxi-tolueno (Butyl-hydroxi-toluen).

4 Valores determinados em laboratório (LANA-UEM, Maringá - PR) (Values determined at Laboratory [LANA-UEM, Maringá-PR]).

5 De acordo com Pezzato et al. (2002) (According to Pezzato et al. [2002]).

6 Valores determinados no laboratório Cromalimentos - DQI-UEM; Maringá-

PR (Values determined at Cromalimentos Laboratory - DQI-UEM, Maringá-PR). 
À dieta foram aspergidos $2 \%$ do produto comercial LUTA-CLA ${ }^{\circledR}$ (BASF do Brasil), composto de $60 \%$ dos isômeros (cis-9,trans-11 e trans-10,cis-12) e 40\% do veículo (oléico e outros ácidos graxos). A dieta foi distribuída duas vezes/dia, às 8 e $17 \mathrm{~h}$. $\mathrm{O}$ arraçoamento foi manual e até saciedade aparente, quando não se observou mais a captura e a regurgitação dos grânulos pelos peixes.

Ao início do experimento, foram retirados filés de cinco peixes para determinação da composição inicial de ácidos graxos dos filés. A cada sete dias, foram coletados ao acaso, utilizando-se puçás, dois peixes de cada unidade experimental. Os peixes foram pesados e sacrificados por meio de superdosagem de xilocaína $(10 \mathrm{~g} / \mathrm{L})$ para retirada dos filés.

Os filés foram mantidos em freezer $\left(-24^{\circ} \mathrm{C}\right)$ para posterior análise do perfil de ácidos graxos. As amostras de cada unidade experimental foram trituradas em liquidificador até obtenção de uma polpa homogênea. Alíquotas desta polpa foram utilizadas para extração e quantificação dos lipídios totais, segundo metodologia de Bligh \& Dyer (1959).

As análises do perfil de ácidos graxos foram realizadas no laboratório Cromalimentos do Departamento de Química da Universidade Estadual de Maringá. Na realização da transesterificação, os lipídios foram submetidos aos processos de metilação, conforme método 5509 da ISO (1978). Os ésteres de ácidos graxos foram analisados por meio do cromatógrafo gasoso 14-A (Shimadzu, J apão), equipado com detector de ionização de chama e coluna capilar de sílica fundida ( $50 \mathrm{~m}$ de comprimento e $0,25 \mathrm{~mm}$ de diâmetro interno). A fase estacionária utilizada foi a Carbowax $20 \mathrm{M}$, fase polar de polietilenoglicol, com um filme de $0,20 \mathrm{~mm}$. Os fluxos dos gases ultrapuros (White Martins) foram de $1,2 \mathrm{~mL} /$ minuto para o gás de arraste $\left(\mathrm{H}_{2}\right) ; 30 \mathrm{~mL} /$ minuto para o gás auxiliar (make-up) $\left(\mathrm{N}_{2}\right)$; e 30 e $300 \mathrm{~mL} /$ minuto para os gases de chama, $\mathrm{H}_{2}$ e ar sintético, respectivamente. A razão de divisão (split) da amostra foi de 1:100. A temperatura da coluna foi de $150^{\circ} \mathrm{C}$ por 5 minutos, sendo, então, elevada para $240^{\circ} \mathrm{C}$ a uma taxa de $2^{\circ} \mathrm{C} /$ minuto. As temperaturas do injetor e detector foram de $220 \mathrm{e} 245^{\circ} \mathrm{C}$, respectivamente. As áreas dos picos foram determinadas por meio do IntegradorProcessador CG-300 (Instrumentos Científicos CG) e a identificação dos picos, pela comparação dos tempos de retenção aos padrões de ésteres metílicos de ácidos graxos (Sigma, EUA).

A taxa de deposição de CLA foi determinada pela função de Gompertz ( $\mathrm{y}=\mathrm{a} \cdot \exp \{-\mathrm{b} \cdot \exp [-\mathrm{kt}]\})(\mathrm{SAS}$, 1995). O meio-tempo (T) para deposição de 50\% do CLA foi calculado pela equação $T=\ln 2 / \mathrm{k}$, em que: $\ln$ é logaritmo Neperiano; e $k$ a velocidade com que a deposição de CLA atinge seu valor máximo no tecido muscular de tilápiasdo-nilo.

\section{Resultados e Discussão}

Como demonstrado nas Figuras 1, 2 e 3, para ambos os isômeros ( cis-9,trans-11 etrans-10,cis-12), foi observada rápida taxa de deposição até o $28^{\circ}$ dia de coleta; após esse período, ocorreu redução na taxa de incorporação de CLA nos filés. Pelo modelo proposto, o tempo decorrido para deposição de CLA para os isômeros cis-9,trans-11 e trans-10,cis-12 foi de 8,26 e 5,19 dias, respectivamente, o que reflete diferenças quanto à velocidade de incorporação desses isômeros nos filés, mesmo quando em valores percentuais iguais nas dietas $(0,6 \%$ cada) .

Estudos realizados com isótopos estáveis têm demonstrado que a taxa de substituição de carbono varia em cada espécie animal e tecido analisado (Kennedy \& Krouse, 1989). Segundo Tieszen et al. (1983), os tecidos que apresentam rápido metabolismo refletem a alimentação recente, enquanto os de baixa taxa de substituição representam alimentações de um período mais prolongado. Segundo Libby et al. (1964), tecidos metabolicamente mais ativos, como o fígado, o pâncreas e o tecido gorduroso têm taxa de substituição mais rápida que os menos ativos, como os ossos e o tecido conjuntivo. Carrijo et al. (2000) observaram essas evidências quando avaliou oturnover de diferentes tecidos de poedeiras comerciais pela técnica dos isótopos estáveis $\left({ }^{13} \mathrm{C} /{ }^{12} \mathrm{C}\right)$.

A taxa de deposição também pode ser influenciada pela taxa de assimilação dos diferentes nutrientes da dieta (Kennedy \& Krouse, 1989). Em peixes, além dos fatores relacionados à qualidade nutricional da dieta, diversos fatores físicos e químicos da água podem influenciar direta ou indiretamente o crescimento dos peixes. A temperatura influencia a taxa metabólica dos animais e está diretamente relacionada ao consumo de alimentos pelos peixes.

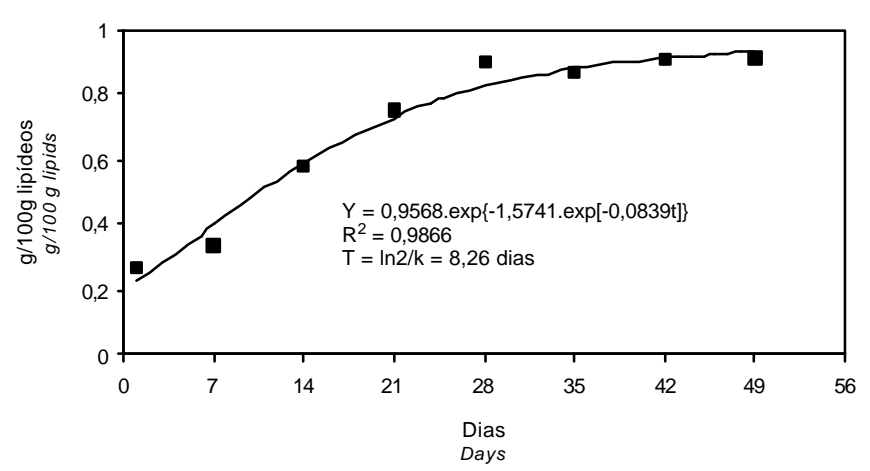

Figura 1 - Função de Gompertz para deposição do isômero cis-9, trans-11 CLA nos lipídios totais em filés de tilápiasdo-nilo.

Figure 1 - Gompertz function for deposition of cis-9, trans-11 isomer CLA in the total lipids of Nile tilapia fillets. 


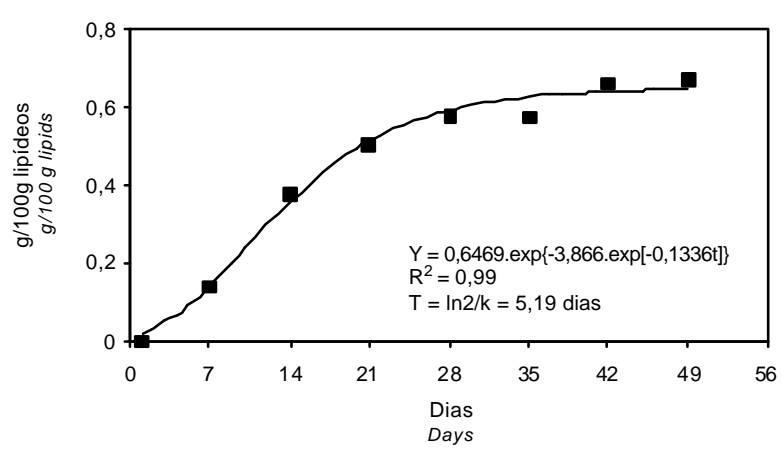

Figura 2 - Função de Gompertz para deposição do isômero trans-10,cis-12 CLA nos lipídios totais de filés de tilápias-do-nilo.

Figure 2 - Gompertz function for deposition of trans-10,cis-12 isomer CLA in the total lipids of Nile tilapia fillets.

A taxa de incorporação também pode ser influenciada pela idade do animal. De acordo com Kennedy \& Krouse (1989), a taxa pela qual os nutrientes são incorporados e eliminados em determinado compartimento biológico deve ser avaliada em animais no estado de equilíbrio, caracterizado por animais adultos, visto que, em animais jovens, é difícil distinguir a incorporação de novos tecidos do material do tecido em crescimento. No entanto, animais utilizados para produção de carne geralmente não se encaixam nessa categoria se considerada a baixa viabilidade econômica de sua exploração.

Neste estudo, a concentração de CLA no músculo de tilápias-do-nilo alimentadas com 1,2\% de CLA foi de 1,61 g/100 g de ácidos graxos. Valores superiores de CLA no músculo foram encontrados por Choi et al. (1999), que observaram em carpa, em tilápias-do-nilo e "rockfish" alimentados com 1,0\% de CLA na dieta concentrações de CLA de 13,3; 4,1 e 5,1 g/100 g de ácidos graxos, respectivamente. A diferença nos valores encontrados nos experimentos pode ser explicada pela variação no produto utilizado ou pelas diferentes idades dos peixes utilizados nos estudos. Em híbridos de "striped bass" (Morone saxatilis) alimentados com 1,0 g/100 g CLA, a concentração de CLA no músculo foi de $8,1 \mathrm{~g} / 100 \mathrm{~g}$ de ácidos graxos (Twibell et al., 2000). Em juvenis de perca-amarela alimentados com 0,5 e 1,0 g/100 g CLA, foram obtidas no músculo concentrações de CLA de 1,26 e 2,92 g/100 g de ácidos graxos, respectivamente (Twibell et al., 2001). Esses dados indicam que a habilidade dos peixes em acumular CLA varia entre espécies e entre concentrações de CLA e de lipídios na dieta (Twibell et al., 2001).

Os produtos lácteos e a carne de ruminantes apresentam valores baixos de CLA (Chin et al., 1992). Em animais que produzem naturalmente o CLA, a soma dos valores dos

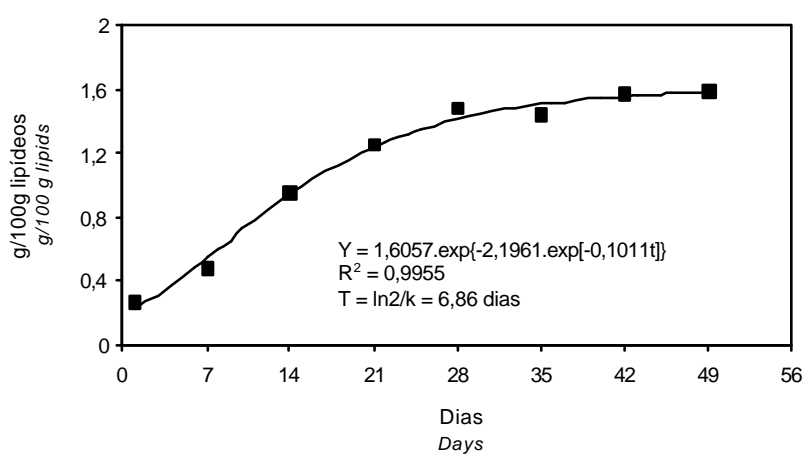

Figura 3 - Função de Gompertz para deposição dos isômeros cis-9,trans-11 e trans-10,cis-12 CLA nos lipídios totais de filés de tilápias-do-nilo.

Figure 3 - Gompertz function for deposition of cis-9,trans -11 and trans-10,cis-12 isomers CLA in the total lipids of Nile tilapia fillets.

isômeros representa de 0,27 a 0,56 g/100 g de ácidos graxos; a gordura do queijo e do leite, por exemplo, contém 0,3 a 0,65 g de CLA/100 g de ácidos graxos. Em comparação a outros animais estudados, peixes possuem grande capacidade de acumular CLA.

A utilização de CLA em dietas para tilápias-do-nilo resultou em alteração na composição de ácidos graxos no músculo (Tabela 2). Ao longo do período experimental, houve aumento linear $(\mathrm{P}<0,01)$ do total de AGPI e dos $n-6$ e diminuição dos AGMI. As alterações encontradas para os ácidos graxos individuais estão relacionadas à diminuição linear $(\mathrm{P}<0,01)$ nas concentrações dos ácidos graxos monoinsaturados 16:1n-7 (palmitoléico), 18:1n-9 (oléico).

Também foram observados aumentos lineares $(\mathrm{P}<0,01)$ nas concentrações dos ácidos graxos 18:2n-6 (linoléico), 22:5n-3 (DPA) e 20:3 n-9. No entanto, o desvio dos dados aumentou nas últimas coletas, o que pode estar relacionado ao método de coleta dos peixes (com puçás), o qual comprometeu o consumo de alimento pelos peixes no período final do experimento. Além disso, ainda que os tanques tenham sido sifonados diariamente e cobertos com lona para evitar a eutrofização, verificou-se a presença de algas no período final do experimento, as quais podem ter contribuído em maior parcela na alimentação dos peixes neste período, acarretando diferença de acúmulo de alguns ácidos graxos das séries n-3 e n-6.

Berge et al. (2004), em estudo com salmão-do-atlântico (Salmo salar), também observaram aumento na concentração dos AGMI 16:1n-7, 18:1n-9, 18:1 n-7 e aumento do 18:0. Resultados semelhantes foram observados por Twibell et al. (2001), que, ao fornecerem CLA em dietas para percaamarela (Perca flavescens), observaram que, em peixes alimentados com dietas com 1,0 g/100 g de CLA, as concentrações de 18:0 e CLA foram aumentadas. Esses autores 
Tabela 2 - Composição de ácidos graxos ( $\mathrm{g} / 100 \mathrm{~g}$ ) no filé da tilápias-do-nilo alimentadas com dietas com 1,2\% de CLA durante 49 dias Table 2 - Fatty acids composition ( $\mathrm{g} / 100 \mathrm{~g}$ ) in the Nile tilapia fillet fed diets with $1.2 \%$ of CLA during 49 days

\begin{tabular}{|c|c|c|c|c|c|c|c|c|}
\hline \multirow[t]{2}{*}{$\begin{array}{l}\text { Ácido graxo } \\
\text { Fatty acid }\end{array}$} & \multicolumn{8}{|c|}{$\begin{array}{c}\text { Tempo (dias) } \\
\text { Time (days) }\end{array}$} \\
\hline & 0 & 7 & 14 & 21 & 28 & 35 & 42 & 49 \\
\hline 14:0 & $2,47 \pm 0,31$ & $2,69 \pm 0,14$ & $2,20 \pm 0,26$ & $1,85 \pm 0,31$ & $2,23 \pm 0,19$ & $1,91 \pm 0,26$ & $2,31 \pm 0,38$ & $1,90 \pm 0,38$ \\
\hline $16: 0$ & $24,76 \pm 0,07$ & $26,36 \pm 4,05$ & $23,15 \pm 1,00$ & $22,16 \pm 0,73$ & $24,20 \pm 0,54$ & $22,82 \pm 1,88$ & $25,78 \pm 0,65$ & $23,66 \pm 1,88$ \\
\hline $16: 1 n-9$ & $0,59 \pm 0,04$ & $0,61 \pm 0,03$ & $0,61 \pm 0,03$ & $0,51 \pm 0,08$ & $0,55 \pm 0,07$ & $0,63 \pm 0,12$ & $0,67 \pm 0,06$ & $0,58 \pm 0,05$ \\
\hline $16: 1 \mathrm{n}-7^{\mathrm{a}}$ & $3,58 \pm 0,87$ & $3,54 \pm 0,62$ & $2,89 \pm 0,46$ & $2,78 \pm 0,69$ & $2,72 \pm 0,40$ & $2,65 \pm 0,23$ & $2,43 \pm 0,32$ & $2,19 \pm 0,42$ \\
\hline $17: 0$ & $0,39 \pm 0,00$ & $0,31 \pm 0,05$ & $0,41 \pm 0,00$ & $0,88 \pm 0,44$ & $0,52 \pm 0,11$ & $0,44 \pm 0,29$ & $0,49 \pm 0,07$ & $0,40 \pm 0,03$ \\
\hline $17: 1 \mathrm{n}-9$ & $0,41 \pm 0,02$ & $0,27 \pm 0,00$ & $0,26 \pm 0,04$ & $0,74 \pm 0,46$ & $0,41 \pm 0,15$ & $0,41 \pm 0,16$ & $0,28 \pm 0,04$ & $0,25 \pm 0,01$ \\
\hline $18: 0$ & $9,61 \pm 2,20$ & $8,71 \pm 1,02$ & $9,56 \pm 0,14$ & $9,93 \pm 0,74$ & $9,06 \pm 0,28$ & $9,26 \pm 0,63$ & $9,68 \pm 1,04$ & $10,24 \pm 0,39$ \\
\hline $18: 1 n-9^{b}$ & $28,37 \pm 2,02$ & $28,33 \pm 0,12$ & $27,24 \pm 2,22$ & $25,39 \pm 1,88$ & $27,33 \pm 0,63$ & $24,98 \pm 1,24$ & $21,62 \pm 1,57$ & $24,08 \pm 1,63$ \\
\hline $18: 1 \mathrm{n}-7$ & $2,85 \pm 0,21$ & $2,78 \pm 0,02$ & $3,06 \pm 0,10$ & $3,63 \pm 1,21$ & $2,52 \pm 0,17$ & $2,78 \pm 0,31$ & $2,78 \pm 0,28$ & $2,56 \pm 0,07$ \\
\hline $18: 1 n-6$ & $0,18 \pm 0,02$ & $0,14 \pm 0,06$ & $0,12 \pm 0,01$ & $0,18 \pm 0,14$ & $0,19 \pm 0,06$ & $0,17 \pm 0,02$ & $0,18 \pm 0,05$ & $0,15 \pm 0,02$ \\
\hline $18: 2 n-6^{c}$ & $13,90 \pm 0,53$ & $14,77 \pm 0,07$ & $14,70 \pm 1,16$ & $17,51 \pm 0,81$ & $16,47 \pm 1,19$ & $16,41 \pm 0,66$ & $16,71 \pm 1,40$ & $17,88 \pm 1,26$ \\
\hline $18: 3 n-6$ & $0,54 \pm 0,28$ & $0,61 \pm 0,07$ & $0,77 \pm 0,03$ & $0,62 \pm 0,04$ & $0,64 \pm 0,03$ & $55 \pm 0,19$ & $0,60 \pm 0,12$ & $0,56 \pm 0,03$ \\
\hline $18: 3 n-3$ & $0,68 \pm 0,06$ & $0,71 \pm 0,03$ & $0,71 \pm 0,10$ & $0,66 \pm 0,09$ & $0,64 \pm 0,13$ & $0,62 \pm 0,06$ & $0,62 \pm 0,15$ & $0,62 \pm 0,04$ \\
\hline $18: 2(9-c, 11-t)$ & $0,24 \pm 0,04$ & $0,35 \pm 0,02$ & $0,60 \pm 0,02$ & $0,50 \pm 0,27$ & $1,06 \pm 0,13$ & $0,80 \pm 0,15$ & $0,90 \pm 0,46$ & $1,02 \pm 0,12$ \\
\hline $18: 2(10-t, 12-c)$ & $0,08 \pm 0,03$ & $0,15 \pm 0,00$ & $0,39 \pm 0,03$ & $0,52 \pm 0,08$ & $0,73 \pm 0,13$ & $0,56 \pm 0,12$ & $0,71 \pm 0,40$ & $0,79 \pm 0,14$ \\
\hline $20: 2 n-9$ & $0,14 \pm 0,05$ & $0,10 \pm 0,01$ & $0,20 \pm 0,08$ & $0,15 \pm 0,02$ & $0,13 \pm 0,02$ & $0,15 \pm 0,03$ & $0,12 \pm 0,02$ & $0,19 \pm 0,05$ \\
\hline $21: 0$ & $0,77 \pm 0,03$ & $0,69 \pm 0,31$ & $1,08 \pm 0,27$ & $96 \pm 0,30$ & $0,95 \pm 0,14$ & $0,96 \pm 0,07$ & $0,92 \pm 0,10$ & $0,94 \pm 0,19$ \\
\hline $20: 2 n-6$ & $0,96 \pm 0,11$ & $0,79 \pm 0,30$ & $1,16 \pm 0,24$ & $0,96 \pm 0,28$ & $0,89 \pm 0,08$ & $1,02 \pm 0,13$ & $0,90 \pm 0,15$ & $0,82 \pm 0,13$ \\
\hline $20: 4 n-6$ & $2,14 \pm 0,35$ & $1,91 \pm 0,68$ & $2,71 \pm 0,40$ & $2,93 \pm 0,82$ & $2,27 \pm 0,21$ & $4,60 \pm 0,97$ & $3,42 \pm 2,16$ & $3,55 \pm 0,55$ \\
\hline $20: 5 n-3$ & $0,13 \pm 0,03$ & $0,11 \pm 0,03$ & $0,17 \pm 0,01$ & $0,13 \pm 0,01$ & $0,13 \pm 0,04$ & $0,16 \pm 0,03$ & $0,22 \pm 0,06$ & $0,18 \pm 0,05$ \\
\hline $22: 4 n-6$ & $1,01 \pm 0,07$ & $0,83 \pm 0,33$ & $1,30 \pm 0,28$ & $1,07 \pm 0,30$ & $1,05 \pm 0,24$ & $1,34 \pm 0,26$ & $1,28 \pm 0,08$ & $1,15 \pm 0,31$ \\
\hline $22: 5 n-6$ & $1,53 \pm 0,29$ & $1,26 \pm 0,58$ & $1,89 \pm 0,19$ & $1,53 \pm 0,68$ & $1,25 \pm 0,24$ & $1,93 \pm 0,42$ & $2,15 \pm 0,43$ & $1,61 \pm 0,24$ \\
\hline $22: 5 n-3^{d}$ & $0,68 \pm 0,07$ & $0,62 \pm 0,27$ & $0,91 \pm 0,30$ & $0,78 \pm 0,18$ & $0,75 \pm 0,21$ & $0,92 \pm 0,08$ & $0,97 \pm 0,23$ & $1,06 \pm 0,38$ \\
\hline $22: 6 n-3$ & $2,50 \pm 0,38$ & $1,98 \pm 0,88$ & $2,48 \pm 0,81$ & $2,19 \pm 0,86$ & $1,62 \pm 0,18$ & $2,58 \pm 0,62$ & $2,67 \pm 0,50$ & $2,30 \pm 0,55$ \\
\hline $20: 3 n-9^{e}$ & $0,09 \pm 0,02$ & $0,12 \pm 0,02$ & $0,12 \pm 0,00$ & $0,13 \pm 0,01$ & $0,13 \pm 0,04$ & $0,15 \pm 0,02$ & $0,16 \pm 0,04$ & $0,16 \pm 0,02$ \\
\hline $\mathrm{AGPI}^{\mathrm{f} *}$ & $24,80 \pm 1,46$ & $24,42 \pm 3,17$ & $28,23 \pm 3,42$ & $29,91 \pm 0,59$ & $27,62 \pm 0,41$ & $31,96 \pm 2,82$ & $31,62 \pm 1,95$ & $32,04 \pm 2,63$ \\
\hline $\begin{array}{l}P U F A \\
\text { AGMI }^{\mathrm{g}}\end{array}$ & $36,70 \pm 1,91$ & $36,38 \pm($ & $34.89 \pm 2$ & 33,8 & 34 & & & \\
\hline$M U F A$ & & & & & & & & \\
\hline AGS & $38,50 \pm 3,37$ & $39,20 \pm 2,77$ & $36,88 \pm 0,73$ & $36,27 \pm 0,64$ & $36,93 \pm 0,51$ & $35,80 \pm 1,57$ & $39,77 \pm 0,42$ & $37,58 \pm 0,40$ \\
\hline$S F A$ & & & & & & & & \\
\hline$n-6^{h}$ & 20,4 & 20,4 & 22 & $=0,98$ & 22 & 26 & 25 & 25 \\
\hline$n-3$ & $3,99 \pm 0,44$ & $3,41 \pm 1,22$ & $4,26 \pm 1,20$ & $3,76 \pm 0,21$ & $3,10 \pm 0,31$ & $4,29 \pm 0,81$ & $4,48 \pm 0,89$ & $4,17 \pm 0,73$ \\
\hline AGPI/AGS & $0,65 \pm 0,09$ & $0,63 \pm 0,13$ & $0,77 \pm 0,11$ & $0,82 \pm 0,03$ & $0,75 \pm 0,02$ & $0,90 \pm 0,11$ & $0,80 \pm 0,05$ & $0,86 \pm 0,07$ \\
\hline$n-6 / n-3$ & $5,14 \pm 0,29$ & $6,28 \pm 1,65$ & $5,50 \pm 1,02$ & $6,90 \pm 0,189$ & $7,35 \pm 0,97$ & $6,17 \pm 0,74$ & $5,79 \pm 1,25$ & $6,46 \pm 1,26$ \\
\hline
\end{tabular}

$\mathrm{a}, \mathrm{b}, \mathrm{c}, \mathrm{d}, \mathrm{e}, \mathrm{f}, \mathrm{g}, \mathrm{h}$ Efeito linear (linear effect) $(\mathrm{P}<0,01): \mathrm{Y}=3,5082-0,0268 \mathrm{x}, \mathrm{R}^{2}=0,92 ; \mathrm{Y}=28,8171-0,1193 \mathrm{x}, \mathrm{R}^{2}=0,68 ; \mathrm{Y}=14,4438+0,0662 \mathrm{x}, \mathrm{R}^{2}=0,67$ $Y=0,6374+0,0079 x, R^{2}=0,77 ; Y=0,0964+0,0014 x, R^{2}=0,94 ; Y=24,9664+0,1594 x, R^{2}=0,80 ; Y=37,3030-0,1593 x, R^{2}=0,85 ; Y=20,8332+0,1156 x$, $\mathrm{R}^{2}=0,73$; respectivamente (respectively).

*AGPI (PUFA) = soma dos ácidos graxos poliinsaturados (sum of polyunsaturated fatty acids); AGMI = soma dos ácidos graxos monoinsaturados (sumofmonounsaturated fatty acids); $\mathrm{AGS}=$ soma dos ácidos graxos saturados (sum of saturated fatty acids); $\mathrm{n}-6$ = soma dos ácidos graxos da série $\mathrm{n}-6$ (sum of $n-6$ serie fatty acids); $\mathrm{n}-3$ = soma dos ácidos graxos n-3 (sum of $n-3$ serie fatty acids); AGPI/AGS = relação entre ácidos graxos polinsaturados e saturados (polyunsaturated: saturated fatty acids ratio); $n-6 / n-3$ = relação entre os ácidos graxos das séries $n-6$ e n-3 (n-6:n-3 ratio).

Médias seguidas de letras diferentes, na mesma linha diferem $(P<0,05)$ pelo teste $t$

Means followed by different letters within a row differ $(P>0.05)$ by the $t$ test.

verificaram diminuição na concentração de 16:1(n-7) e 18:1(n-7) e aumento na concentração total de AGPI n-6 nos lipídios do músculo de peixes alimentados com dietas com $0,5 \mathrm{~g} / 100 \mathrm{~g}$ de CLA. Twibell et al. (2000), em estudo realizado com híbrido de "striped bass" alimentado com 0,5 a 1,0\% de CLA, também notaram aumento do 18:0 e diminuição do 18:1n-7 nos filés dos peixes.

Yasmin et al. (2004), estudando juvenis de tilápia-donilo alimentados com dieta com 5,0\% de CLA, observaram diminuição do ácido linoléico (18:2n-6) e do total de n-3, do DHA (22: 6 n-3) e dos AGPI, assim como alterações nos níveis de ácido araquidônico (20:4 n-9) com o fornecimento de CLA, o que difere dos resultados obtidos nesse experimento para tilápia-do-nilo. A redução na concentração de AGMI observada por Yasmin et al. (2004) está de acordo com os resultados encontrados por Twibell et al. (2000).

Lee et al. (1998) sugeriram que o isômero trans-10, cis-12 CLA reduz a relação AGPI por AGS, inibindo a atividade da enzima stearoyl-coenzima A dessaturase ( $\Delta 9)$ no fígado, que cataliza a inserção de uma dupla ligação entre os átomos C9 e C10, tanto no 16:0 quanto no 18:0, para a formação do 16:1 (n-7) e 18:1 (n-9), respectivamente. Desse modo, o fornecimento de CLA com a presença desse isômero acarreta a inibição da enzima $\Delta 9$ dessaturase, resultando 
em acúmulo de ácidos graxos saturados, os quais conduzem a uma mudança da composição dos ácidos graxos nos tecidos dos animais (Watkins et al., 2003).

A produção de alimentos funcionais tem se tornado uma importante área de estudo e a ingestão de CLA como um alimento funcional está estreitamente relacionada à prevenção de câncer e arteriosclerose em humanos, assim como à melhora no sistema imunológico e à redução na deposição de lipídio corporal. Assim, é importante a realização de estudos sobre a taxa de deposição de CLA em produtos destinados à alimentação humana. Os resultados deste estudo confirmam que o CLA pode ser incluído em dietas para tilápia-do-nilo objetivando sua incorporação nos lipídios totais do filé.

\section{Conclusões}

A utilização de CLA durante 30 dias antes do abate é suficiente para permitir sua adequada deposição nos lipídios totais do filé. O fornecimento de CLA diminui a concentração de AGMI e aumenta a concentração de AGS nos lipídios totais de filés de tilápia-do-nilo.

\section{Agradecimento}

À BASF do Brasil, pelo apoio científico, e à CAPES (Coordenação de Aperfeiçoamento de Pessoal de Nível Superior), pela concessão de bolsa de doutorado ao primeiro autor.

\section{Literatura Citada}

ACKMAN, R.G. Conjugated linoleic acid (CLA) in lipids of fish tissues. In: YURAWECZ, M.P.; MOSSOBA, M.M.; KRAMER, J.K.G. et al. (Eds.) Advances in conjugated linoleic acid research. Champaign: AOCS Press, 1999. v.1, p.283-295.

BERGE, G.M.; RUYTER, B.; ASGARD, T. Conjugated linoleic acid in diets for juvenile Atlantic salmon (Salmo salar); effects on fish performance, proximate composition, fatty acid and mineral content. Aquaculture, v. 237, p.365-380, 2004.

BLIGH, E.G.; DYER, W.J. A rapid method of total lipid extraction and purification. Canadian Journal of Biochemistry, v.37, n.18, p.911-917, 1959.

CARRIJO, A.S.; PEZZATO, A.C.; DUCATTI, C. Avaliação do metabolismo nutricional em poedeiras pela técnica dos isótopos estáveis do carbono $\left({ }^{13} \mathrm{C} /{ }^{12} \mathrm{C}\right)$. Revista Brasileira de Ciência Avícola, v.2, n.3, p.209-218, 2000.

CHIN, S.F.; LIU, W.; STORKSON, J.M. et al. Dietary sources of conjugated dienoic isomers of linoleic acid a newly ecognized class of anticarcinogens. Journal of Food Composition and Analysis, v.5, p.185-197, 1992.
INTERNATIONAL ORGANIZATION FOR STANDARDIZATION ISO. Animal and vegetable fats and oils preparation of methyl esters of fatty acids. Geneve: ISO. Method ISO 5509, 1978. p.1-6.

KENNEDY, B.V.; KROUSE, H.R. Isotope fractionation by plants and animals: implications for nutrition research. Canadian Journal Physiology and Pharmacology, v.68, p.960-972, 1989.

LEE, K.N.; PARIZA, M.W.; NTAMBI, J.M. Conjugated linoleic acid decreases hepatic stearoyl-CoA desaturase mRNA expression. Biochemical and Biophysical Research Communications, v. 248, p. $817-821,1998$

LIBBY, W.F.; BERGE, R.; MEAD, J. Replacement rates for human tissue from atmospheric radiocarbon. Science, v.146, p.1170$1172,1964$.

OSTROWSKA, E.; MURALITHARAN, M.; CROSS, R.F. et al. Dietary conjugated linoleic acids increase lean tissue and decrease fat deposition in growing pigs. Journal of Nutrition, v.129, p.2037-2042, 1999.

PARIZA, M.W.; PARK, Y.; COOK, M.E. The biologically active isomers of conjugated linoleic acid. Progress in Lipid Research, v.40, p.283-298, 2001.

PARK, Y.; ALBRIGHT, K.J.; LIU, W. et al. Effect of conjugated linoleic acid on body composition in mice. Lipids, v.32, 853858, 1997.

PEZZATO, L.E.; MIRANDA, E.C.; PEZZATO, A.C. et al. Digestibilidade aparente de ingredientes pela tilápia do Nilo (Oreochromis niloticus). Revista Brasileira de Zootecnia, v.31, n.4, p.1595-1604, 2002.

STATISTICAL ANALYSIS SYSTEMS - SAS. User's guide. version 6.08. Cary: 1995. 846p

TIERSZEN, L.L.; BOUTTON, T.W.; TESDAHL, K.G. et al. Fractionation and turnover of stable carbon isotopes in animal tissues: implications for $\delta^{13} \mathrm{C}$ analysis of diet. Oecologia, v.57, p.32-37, 1983.

TWIBELL, R.G.; WATKINS, B.A.; BROWN, P.B. Dietary conjugated linoleic acids and lipid source alter fatty acid composition of juvenile yellow perch, Perca flavescens. Journal of Nutrition, v.131, p.2322-2328, 2001.

TWIBELL, R.G.; WATKINS, B.A.; ROGERS, L. et al. Effects of dietary conjugated linoleic acids on hepatic and muscle lipids in hybrid striped bass. Lipids, v.35, p.155-161, 2000.

WATKINS, B.A.; FENG, S.; STROM, A.K. et al. Conjugated linoleic acids alter the fatty acid composition and physical properties of egg yolk and albumen. Journal of Agricultural and Food Chemistry, v.51, p.6870-6876, 2003.

WHIGHAM, L.D.; COOK, M.E.; ATKINSON, R.L. Conjugated linoleic acid: implications for human health. Pharmacological Research, v.42, n.6, p.503-510, 2000.

YANG. L.; HUANG, Y.; JAMES, A.E. et al. Differential incorporation of conjugated linoleic acid isomers into the egg yolk lipids. Journal of Agricultural and Food Chemistry, v.50, p.4941-4946, 2002.

YASMIN, A.; TAKEUCHI, T.; HAYASHI, M. et al. Effect of conjugated linoleic acid and docosahexanoic acids on growth of juvenile Oreochromis niloticus. Fisheries Science, v.70, p.473-481, 2004. 